\title{
HÁBITOS ALIMENTARIOS, ACTIVIDAD FÍSICA Y SU RELACIÓN CON EL ESTADO NUTRICIONAL-ANTROPOMÉTRICO DE PREESCOLARES
}

\section{ANTHROPOMETRIC-NUTRITIONAL ASSESSMENT, DIETARY HABITS AND PHYSICAL ACTIVITY IN PRESCHOOL CHILDREN}

\author{
Mariné Coromoto Nava B. (1), Analy Pérez G. (2), \\ Héctor Antonio Herrera (2), Rosa Armenia Hernández H. (3) \\ (1) Hospital Militar "Dr. Carlos Arvelo" \\ Departamento de Nutrición y Dietética. Caracas, Venezuela. \\ (2) Universidad Simón Bolívar \\ Departamento de Tecnología de Procesos Biológicos y Bioquímicos, Caracas, Venezuela. \\ (3) Universidad Simón Bolívar \\ Laboratorio de Evaluación Nutricional. Caracas, Venezuela.
}

\begin{abstract}
Preschool Venezuelan children underwent a study that measures the relationship between anthropometric nutritional state, food habits and physical activity level (PAL). Methods: Using Frisancho's pattern as a reference, 173 children were studied, and anthropometric indicators such as height/age, weight/height and both lean and fat arm areas were measured. To assess food habits and PAL, a questionnaire was developed and applied. Results: For vegetables, fruits and fats the consumption patterns was low, and for cereals, meats, and dairy products was high. In $52 \%$ of the children the PAL was intense, and in the $27 \%$ was light. Among food habits, PAL and sedentary index (SI) an association was found. Conclusions: Results suggest implementing changes from the preschool stage to correct for inappropriate food habits, to encourage physical activity and to avoid a high SI, will relate to the appearance of adult chronic degenerative diseases.

Key words: preschool children, anthropometric nutritional assessment, dietary habits, physical activity.
\end{abstract}

Este trabajo fue recibido el 8 de Junio de 2010, aceptado con modificaciones el 1 de Noviembre de 2010 y aceptado para ser publicado el 7 de Junio de 2011.

\section{INTRODUCCIÓN}

La edad preescolar se caracteriza por ser una etapa en la que se adquieren los hábitos que van a definir el estilo de vida del futuro adulto (1), es por ello que es pertinente implementar en esta etapa, cualquier acción que permita un crecimiento y desarrollo normal. Algún cambio desfavorable en el estilo de vida del niño, comprometerá su formación biológica, que de una u otra forma afectará su estado nutricional y por ende su salud actual y futura.

Diversos factores influyen en el estado nutricional del niño, entre ellos los hábitos alimentarios y la actividad física, no obstante, pocos son los estudios que relacionan éstas variables en niños de edad preescolar.

En cuanto al estado nutricional, los estudios reali- zados en preescolares venezolanos indican la existencia de ambas condiciones (déficit y exceso). Solano y col. (2), encontraron un $14,3 \%$ de déficit y $20,8 \%$ de exceso nutricional en preescolares de Valencia, mientras que Del Real y col. (3) hallaron un $25,6 \%$ de déficit y 6,4 $\%$ exceso nutricional en preescolares del estado Mérida.

Algunas de las investigaciones que relacionan el estado nutricional del niño con sus hábitos alimentarios y su actividad física, concuerdan en que aquellos que presentan sobrepeso u obesidad, poseen un bajo nivel de actividad física e inadecuados hábitos alimentarios, en comparación con sus similares que se encuentran con un estado nutricional normal (4-5).

Dada que la tendencia actual es al incremento de las prevalencias de sobrepeso u obesidad en niños (6-7) 
y en vista del incremento en la aparición de enfermedades crónicas no trasmisibles del adulto (ECNTA), es importante ejercer acciones en las primeras etapas de la vida tal como lo propone la Organización Mundial de la Salud (OMS) (8) contribuyendo a la adquisición de un adecuado estado nutricional del adulto. El presente estudio tuvo como objetivo conocer la relación entre hábitos alimentarios, actividad física y estado nutricionalantropométrico en preescolares de una comunidad de la Región Capital de Caracas, Venezuela.

\section{SUJETOS Y MÉTODOS}

El estudio fue de tipo transversal, no experimental, descriptivo e intencional no probabilístico. El proyecto fue revisado y aprobado por la Coordinación de Ciencias de los Alimentos y Nutrición de la Universidad Simón Bolívar. Previo al estudio, se cumplieron con los principios de ética reflejados en la declaración de Helsinky de 1964 (9), revisados en 2000, la cual define las pautas éticas para la investigación en seres humanos, cumpliéndose con obtener el consentimiento informado a los padres y representantes. Se evaluaron 173 niños de 4 a 6,9 años de edad, 93 de sexo femenino $(53,8 \%)$ y 80 del sexo masculino $(46,2 \%)$ con un promedio de edad de 5.5 años. Los niños fueron seleccionados en 5 instituciones educativas (3 públicas y 2 privadas) pertenecientes al Distrito Capital, Caracas-Venezuela.

\section{Evaluación nutricional antropométrica}

Las mediciones antropométricas fueron realizadas por la autora del estudio, previo entrenamiento y estandarización, de acuerdo a la metodología propuesta por el Programa Biológico Internacional (10). Las mediciones realizadas fueron peso $(\mathrm{Kg})$, talla $(\mathrm{cm})$, circunferencia media de brazo $(\mathrm{CB})(\mathrm{cm})$ y pliegue de tríceps $(\mathrm{PTr})$ (mm). Se utilizó una balanza portátil digital marca Tanita (con precisión de $0,1 \mathrm{Kg}$ ) para la medición del peso, una cinta métrica y escuadra de madera para medir la talla con el método de la plomada (11), una cinta métrica marca Holtain Limited para las medidas de circunferencia y un calibrador marca Holtain Limited (con una precisión $2 \mathrm{~mm}$ ) para la medición de pliegues cutáneos.

Se estudiaron los indicadores de masa corporal total tales como la talla para la edad (TE) y el peso para la talla (PT); los indicadores de composición corporal como el área magra (AM) y el área grasa (AG), se utilizaron las siguientes ecuaciones:

Área magra del brazo $=(\mathrm{CB}-(\pi \times \mathrm{PTr}))^{2} / 4 \pi \mathrm{y}$ Área Grasa del brazo: $=((\mathrm{CBxPtr}) / 2)-\left(\left(\pi \mathrm{x}(\mathrm{Ptr})^{2}\right) / 4\right)(12)$.

La categorización del estado nutricional de los preescolares se realizó de acuerdo a los valores de puntaje de Z-, según sexo y edad y con los siguientes puntos de corte: bajo la normal lo cual es indicativo de déficit nutricional(<-1DE), normal $( \pm 1 \mathrm{DE})$ y sobre lo normal lo cual indica exceso ( $>1 \mathrm{DE})$, utilizando como mediana de la población de referencia, los valores propuesto por Frisancho (13), ya que para el momento de realizar el estudio no se contaban con los valores de referencia de composición corporal de la OMS.

\section{Evaluación del patrón de consumo alimentario a).- Prueba Piloto}

Para la evaluación del patrón de consumo de alimentos de los niños estudiados, se procedió a elaborar un instrumento de recolección con los datos de consumo, que consistió en la aplicación de un recordatorio de 24 horas (consumo del día anterior a la entrevista) de forma aleatoria, a un total de 20 padres y madres de preescolares entre 4 y 6,9 años de edad, pertenecientes a la comunidad objeto de estudio. La finalidad fue obtener la lista de los alimentos más consumidos, para así diseñar el cuestionario de frecuencia de consumo semanal de alimentos.

\section{b).- Frecuencia de consumo de alimentos:}

Una vez aplicado el cuestionario de frecuencia de consumo de alimentos a la muestra total estudiada, se clasificó en bajo, normal ó alto, para cada grupo de alimentos, de acuerdo a las recomendaciones de raciones de alimentos para niños y niñas de 2 a 6 años del Instituto Nacional de Nutrición de Venezuela (14). El consumo diario considerado como normal para el grupo de cereales y derivados estaría entre 5-8 raciones al día, el de frutas entre 5 a 6 , el de vegetales y lácteos entre 1 a 2 raciones, el de carnes, quesos y huevos entre 3 a 4 raciones y el de grasas entre 5-6 raciones al día.

\section{Evaluación del nivel de actividad física}

El nivel de actividad física fue evaluado a partir del cuestionario desarrollado y validado por Prista y col. (15), el cual se modificó y se adaptó a la población de estudio. En cuanto a la estimación del gasto energético de cada actividad física, se determinó de acuerdo a las tablas de Ainsworth y col. (16); luego se procedió a multiplicar la frecuencia semanal de cada actividad, estimando así el gasto energético de cada una de ellas en equivalentes metabólicos (METs). Posteriormente, se dividió entre el número de horas que el individuo realizaba dicha actividad, obteniéndose el gasto energético por semana y posteriormente por día. Finalmente, se multiplicó por el peso del individuo para obtener la Energía de Actividad (EA) (17), la cual se sumó a la Tasa Metabólica Basal (TMB) y la energía por la Actividad Dinámica Específica (ADE), para la obtención del Gasto Energético Total 
(GET). El GET se relacionó con la TMB y se obtuvo finalmente el Nivel de Actividad Física (NAF), el cual se catalogó en leve, moderado e intenso según sexo y edad de los preescolares, de acuerdo a la clasificación propuesta por Torun y col. (18). Para niñas y niños de 1 a 5 años de edad el NAF normal está entre 1,45 a 1,61; para los niños y niñas mayores de 5 años de edad el NAF normal está entre los rangos 1,55 a 1,95 y 1,47 a 1,85 respectivamente.

Se calculó el índice de sedentarismo (IS) de los niños evaluados de acuerdo a la ecuación propuesta por Martínez y col, (19), a través del cual se determina el promedio de horas en que el niño ve televisión, juega videos ó utiliza la computadora diariamente. El IS no debe ser mayor a 2 horas días de acuerdo a la Academia Americana de Pediatría (20). Además, de acuerdo a su adecuación con las recomendaciones diarias para éste tipo de actividades, mientras más cercano a 1 es el índice, mayor será el nivel de sedentarismo del individuo.

\section{Análisis estadístico}

Para el procesamiento de los datos se utilizó el programa SPSS para Windows versión 13.0. Se calcularon los estadísticos descriptivos básicos (promedios y desviación estándar) para la caracterización de la muestra por edad y sexo.

Para la comparación de resultados se realizó una Análisis de Varianza (ANOVA) en combinación con el Test de Sheffé consideración significación estadística un valor de $\mathrm{p}<0,05$ : La prueba de Chi cuadrado fue utilizada en variables no paramétricas a un nivel de significación del $95 \%$.

Se aplicó un análisis de componentes principales (ACP), con la finalidad de encontrar la asociación entre las variables del estudio (estado nutricional-antropométrico, frecuencia de consumo de alimentos y nivel de actividad física).

\section{RESULTADOS \\ Indicadores antropométricos del estado nutricional}

La caracterización de las variables e indicadores antropométricos en los preescolares por grupos de edad y sexo, se muestra en la tabla 1 . Se observa un incremento en peso y talla con la edad, siendo mayor en el sexo masculino, con excepción del grupo de 5 años de edad, estas diferencias fueron estadísticamente significativas $(\mathrm{p}<0.05)$. En cuanto a los indicadores de composición corporal, los varones presentaron valores ligeramente superiores en las áreas musculares, lo que sugiere un predominio del desarrollo musculo esquelético en los niños; por el contrario en las niñas se observaron valores superiores en las áreas grasas, lo cual sugiere predominio en la acumulación de tejido adiposo en el sexo femenino. Estas diferencias fueron estadísticamente significativas $(\mathrm{p}<0.05)$

En la tabla 2 se presentan los resultados de las prevalencias por categorías de los indicadores antropométricos del estado nutricional. El predominio de individuos dentro de la condición de normalidad es mayor en

\section{TABLA 1}

\section{Caracterización de la valoración nutricional antropométrica en los preescolares, por grupos de edad y sexo.}

\section{4 años \\ 5 años \\ 6 años}

\begin{tabular}{lcccccccccccc}
$\begin{array}{c}\text { Variables } \\
\mathbf{n = 1 7 3}\end{array}$ & \multicolumn{2}{c}{ Femenino } & \multicolumn{2}{c}{ Masculino } & \multicolumn{2}{c}{ Femenino } & Masculino & Femenino & Masculino \\
& $\mathbf{x}$ & $\mathbf{D E}$ & $\mathbf{x}$ & $\mathbf{D E}$ & $\mathbf{x}$ & $\mathbf{D E}$ & $\mathbf{x}$ & $\mathbf{D E}$ & $\mathbf{x}$ & $\mathbf{D E}$ & $\mathbf{x}$ & $\mathbf{D E}$
\end{tabular}

*diferencias estadísticamente significativas $(\mathrm{p}<0,05)$. Test Estadístico ANOVA en combinación con el Test de Sheffé

CMB: Circunferencia media del brazo; PTr: pliegue de tríceps; AM: área magra; AG: área grasa 
comparación con las otras categorías, no obstante, una elevada proporción de la muestra se encontró dentro de la categoría de déficit, con porcentajes que superan lo esperado principalmente para el indicador TE $(31.8 \%)$ para la muestra total, así como también para el indicador AG se encontró un alto porcentaje de niñas bajo la norma o en condición de déficit (18.3\%). Al evaluar las prevalencias del AM y AG para la muestra total, se apreció como la mayoría de los preescolares estudiados (más del 80\%) se ubicaron dentro de los rangos de normalidad. En cuanto a las categorías de déficit y exceso, cabe mencionar que si bien se presentaron prevalencias superiores en la categoría sobre la norma para el componente muscular $(9,8 \%)$ en comparación con la condición de déficit muscular (7\%), por el contrario para el componente graso se apreciaron porcentajes superiores en los niños en el estado de déficit de grasa $(12,1 \%)$, que aquellos varones con exceso de los depósitos de tejido adiposo $(7,5 \%)$.

\section{Hábitos alimentarios}

En cuanto a las prevalencias del consumo de alimentos (tabla 3), los preescolares tuvieron una baja frecuencia en el consumo de frutas, vegetales y grasas, en contraposición el consumo de cereales, carnes y lácteos fue alto en uno y otro género. De los alimentos misceláneos, los de mayor consumo fueron el azúcar y las golosinas (19\% respectivamente).

\section{Nivel de actividad física}

Los preescolares de este estudio presentaron en su mayoría un NAF intenso (tabla 4), especialmente las niñas (55.9\%), y un $27,2 \%$ de la muestra total se categorizó dentro de un NAF leve. En el gráfico I se evidenció que las niñas tienen un menor IS en comparación con los niños, a excepción del grupo de niñas de

\section{TABLA 2}

\section{Prevalencias del estado nutricional según indicadores de masa corporal total y composición corporal para el grupo total por sexo.}

\begin{tabular}{|c|c|c|c|c|c|c|c|c|c|}
\hline \multirow{2}{*}{$\begin{array}{c}\text { Variables } \\
n=173\end{array}$} & \multicolumn{2}{|c|}{ Bajo la norma } & \multirow{2}{*}{$\begin{array}{c}\text { Total } \\
\%\end{array}$} & \multicolumn{2}{|c|}{ Normalidad } & \multirow{2}{*}{$\begin{array}{c}\text { Total } \\
\%\end{array}$} & \multicolumn{2}{|c|}{ Sobre la norma } & \multirow{2}{*}{$\begin{array}{r}\text { Total } \\
\%\end{array}$} \\
\hline & Niñas (\%) & Niños (\%) & & Niñas (\%) & Niños (\%) & & Niñas (\%) & Niños (\%) & \\
\hline $\mathrm{T} / \mathrm{E}$ & 33.3 & 30.0 & 31.8 & 61.3 & 61.3 & 61.3 & 5.4 & 8.8 & 6.9 \\
\hline $\mathrm{P} / \mathrm{T}$ & 14.0 & 8.8 & 11.6 & 65.6 & 83.8 & 74.0 & 20.4 & 7.5 & 14.5 \\
\hline A M & 6.5 & 7.5 & 7.0 & 83.9 & 82.5 & 83.2 & 9.7 & 10.0 & 9.8 \\
\hline A G & 18.3 & 5.0 & 12.1 & 71 & 91.3 & 80.3 & 10.8 & 3.8 & 7.5 \\
\hline
\end{tabular}

T/E: talla para la edad; P/T; peso para la talla; AM: área magra; AG: área grasa

TABLA 3

Prevalencias del consumo de alimentos por sexo

\begin{tabular}{|c|c|c|c|c|c|c|c|c|c|}
\hline \multirow{2}{*}{$\begin{array}{l}\text { Grupos de } \\
\text { alimentos } \\
(n=173)\end{array}$} & \multicolumn{2}{|c|}{ Bajo } & \multirow{2}{*}{$\begin{array}{c}\text { Total } \\
\%\end{array}$} & \multicolumn{2}{|c|}{ Normal } & \multirow{2}{*}{$\begin{array}{c}\text { Total } \\
\%\end{array}$} & \multicolumn{2}{|c|}{ Alto } & \multirow{2}{*}{$\begin{array}{c}\text { Total } \\
\%\end{array}$} \\
\hline & Niñas (\%) & Niños (\%) & & Niñas $(\%)$ & Niños (\%) & & Niñas $(\%)$ & Niños (\%) & \\
\hline Cereales & 33.3 & 31.3 & 32.4 & 24.7 & 27.5 & 26 & 41.9 & 41.3 & 41.6 \\
\hline Frutas & 77.4 & 76.3 & 76.9 & 3.2 & 8.8 & 5.8 & 19.4 & 15.0 & 17.3 \\
\hline Vegetales & 47.3 & 37.5 & 42.8 & 14.0 & 27.5 & 20.2 & 38.7 & 35.0 & 37.3 \\
\hline Lácteos & 32.3 & 18.8 & 26.0 & 29.0 & 41.3 & 34.7 & 38.7 & 40.0 & 39.3 \\
\hline Carnes & 22.6 & 25 & 23.7 & 23.7 & 31.3 & 27.2 & 53.8 & 43.8 & 49.1 \\
\hline Grasas & 83.9 & 82.5 & 83.2 & 8.6 & 8.8 & 8.7 & 7.5 & 8.8 & 8.1 \\
\hline
\end{tabular}


6 años quienes tienen el mayor índice de sedentarismo de todos los preescolares del estudio.

\section{Relación entre estado nutricional-antropométrico, hábitos alimentarios y nivel de actividad física}

En el gráfico II se presenta el análisis de componentes principales de la muestra, en donde se relacionan cada una de las variables del estudio. Se aprecia como dichas variables se distribuyen en dos dimensiones, con lo que se explica el $86,8 \%$ de la varianza total. En la dimensión
1 se observa como los indicadores antropométricos del estado nutricional se encuentran asociados entre sí, tal como se esperaba, presentando una relación débil con el IS. En la dimensión 2 las variables más influyentes son las relacionadas con los hábitos alimentarios, asociándose éstas a su vez con el NAF y el IS.

\section{DISCUSIÓN}

El estado nutricional del individuo está influenciado por múltiples factores, tales como factores ambientales,

\section{TABLA 4}

Valores porcentuales por categorías del nivel de actividad física (NAF) para el grupo total, por edad y sexo.

\begin{tabular}{|c|c|c|c|c|c|c|}
\hline \multirow{2}{*}{$\underset{n=173}{\text { NAF }}$} & \multicolumn{2}{|c|}{ Femenino } & \multicolumn{2}{|c|}{ Masculino } & \multicolumn{2}{|c|}{ Total } \\
\hline & $\mathbf{N}^{\mathbf{o}}$ & $\%$ & $\mathbf{N}^{\mathbf{o}}$ & $\%$ & $\mathbf{N}^{\mathbf{o}}$ & $\%$ \\
\hline Leve & 23 & 25.0 & 24 & 30.0 & 47 & 27.2 \\
\hline Moderado & 18 & 19.4 & 18 & 22.5 & 36 & 20.8 \\
\hline Intenso & 52 & 55.9 & 38 & 47.5 & 90 & 52 \\
\hline
\end{tabular}

\section{GRÁFICO I}

Índice de sedentarismo (IS) de los preescolares.

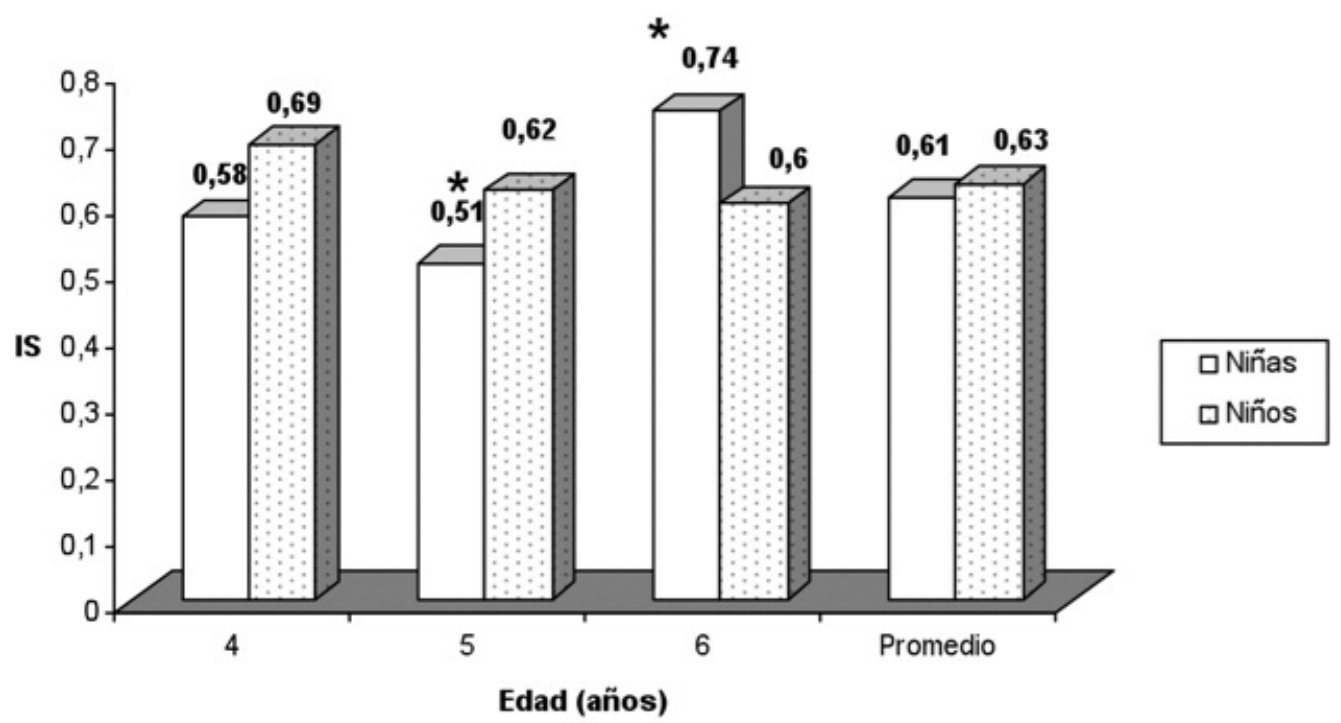

*diferencias estadísticamente significativas $(\mathrm{p}<0,05)$.

Test Estadístico ANOVA en combinación con el Test de Sheffé. 
sociales, económicos, culturales y políticos. Particularmente en niños preescolares, los hábitos alimentarios y la actividad física juegan un papel primordial. Se ha observado que los niños obesos poseen una mayor ingesta de alimentos y un menor NAF, en comparación con sus similares eutróficos (21-23).

En la actualidad, los preescolares tienden a presentar una mayor prevalencia de sobrepeso u obesidad que de déficit nutricional, tal como lo demostraron Kain y cols. en un estudio en preescolares chilenos (24). Sin embargo, estos autores emplearon como indicador del estado nutricional el Indice de Masa Corporal (IMC) únicamente. No obstante, en el presente estudio se utilizaron indicadores de masa y de composición corporal que si bien en preescolares es difícil la toma de las medidas antropométricas para su construcción, resultan ser prácticos y confiables por lo que son ampliamente utilizados para la determinación del estado nutricional en niños.

En ésta investigación se encontró una elevada proporción de niños con déficit según los indicadores de masa corporal total y componente graso, lo que se asemeja a los resultados obtenidos por Del Real (3) en preescolares venezolanos, en donde el déficit fue superior al exceso nutricional por combinación de indicadores. Igualmente el Sistema de Vigilancia Alimentaria y Nutricional venezolano en su último informe (25), reveló que en niños de edad preescolar existía un mayor porcentaje de déficit en contraposición al exceso. No obstante, diversos factores influyen de forma aislada o combinada en el estado nutricional de los niños, por ello, se puede inferir que una de las posibles causas de la elevada prevalencia de niños en déficit nutricional pudiera estar relacionada con sus inadecuados hábitos alimentarios, ya que estos tenían un bajo consumo de frutas, vegetales y particularmente de grasas, cuyos requerimiento son esenciales en ésta etapa, además de conformar una importante fuente de energía y micronutrientes tanto para el crecimiento y el desarrollo. Cabe resaltar, que también los niños estudiados tenían un elevado consumo de carne, lo cual se sabe está relacionado con la aparición de diabetes mellitus tipo II (26). No obstante, no se logró asociar los hábitos alimentarios con el estado nutricional antropométrico (tanto los indicadores de masa como de composición corporal), lo cual se asemeja a lo encontrado por Dawies (27) en un grupo de preescolares europeos, en donde se analizó la relación entre la composición de la dieta (utilizando el método de la pesada de alimentos, el cual es más preciso por ser cuantitativo) y el IMC, sin encontrar asociación; ellos concluyeron que quizás los niveles de actividad

\section{GRÁFICO II}

Distribución de los componentes principales de la muestra.

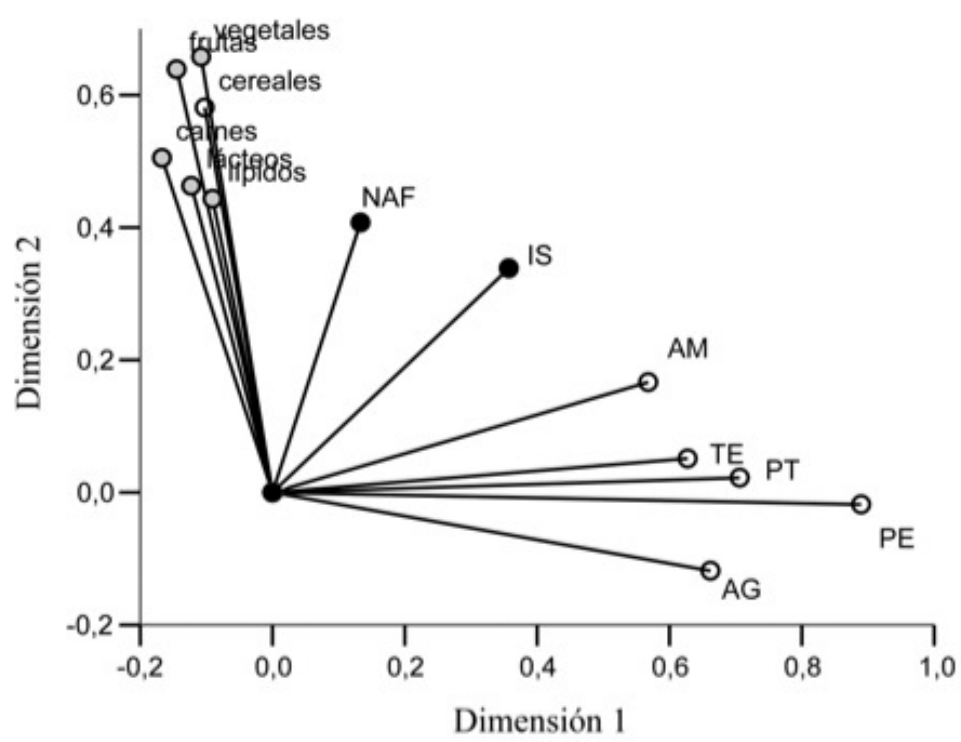

Test estadístico: Análisis de Componentes Principales.

NAF: nivel de actividad física; IS: índice de sedentarismo; TE: talla para la edad; PT; peso para la talla; AM: área magra; AG: área grasa. 
física podrían tener una influencia más importante sobre el IMC en preescolares.

Al evaluar otros de los factores que pudiesen influir en el estado nutricional de los niños, se encontró una relación entre el NAF con los hábitos alimentarios, más no así con el estado nutricional antropométrico, lo que coincide con lo descrito por Fanjiang y Kleinman (28). Estos autores reportan que el primer parámetro que se modifica en el caso de deficiencia energética proveniente de los alimentos, es la actividad física, y la afectación de los parámetros antropométricos se manifiesta en períodos prolongados de restricción energética. Vásquez y cols (29) estudiaron la relación entre balance de energía, composición corporal y NAF en preescolares obesos y eutróficos, utilizando sensores de movimiento y acelerómetros para medir el gasto energético por actividad física y tampoco encontraron relación con el estado nutricional, pero si con la ingesta energética lo cual resulta más evidente en la condición de obesidad. Por otra parte, la mayoría de los preescolares pasaba un alto número de horas viendo televisión al día, lo que se reflejó en un elevado IS, lo cual es considerado como factor obesogénico (30).

Lioret y cols (31), encontraron una relación entre los hábitos alimentarios, el NAF y el estado nutricional, utilizando una metodología similar a la del presente estudio, sin embargo, no utilizaron indicadores de composición corporal que resultan importantes para la determinación a expensas de cual componente corporal se presenta la malnutrición por déficit o exceso.

Al evaluar la falta de asociación entre los indicadores tradicionales de masa corporal como de composición corporal y NAF, se puede inferir que la actividad física no fue lo suficientemente intensa o frecuente para inducir cambios en los componentes corporales, o el tipo de actividad física realizada aunado a los altos IS encontrados, no tuvieron impacto en el incremento de las reservas musculares o deplesión de los depósitos de grasa. Estos hallazgos coinciden con los reportados por Giraldo y cols. (32), que señalan como el instrumento de evaluación de la actividad física puede ser un factor limitante para la evaluación de la misma.

La falta de asociación entre los indicadores antropométricos y el NAF orienta a realizar futuras investigaciones, tales como ahondar la relación entre NAF y consumo de alimentos en preescolares, cuya asociación fue positiva, utilizando instrumentos que permitan la medición de éstas variables en forma no sólo cualitativa sino también cuantitativa.

En éste estudio se refleja como la etapa preescolar podría ser la época ideal en la vida para aplicar medidas preventivas contra la aparición de ECNTA, no sólo porque es una etapa de adquisición de hábitos, sino porque en éstos niños ya se presenta un elevado IS, según lo que se refleja en ésta investigación, que en conjunto con los inadecuados hábitos alimentarios reportados en un alto porcentaje de la muestra evaluada, representan factores de riesgo para la adquisición de éstas enfermedades. Por ello, implementar estrategias para fomentar tanto el incremento del nivel de actividad física, particularmente en el sexo femenino, así como corregir los inadecuados hábitos alimentarios sería beneficioso para evitar la aparición de ECNTA desde etapas tempranas de la vida, y prevenir la aparición de enfermedades cardiovasculares que constituyen la primera causa de morbimortalidad en Venezuela y en muchos otros países latinoamericanos.

\section{RESUMEN}

Se estudió la relación entre el estado nutricional medido por antropometría, los hábitos alimentarios y el nivel de actividad física (NAF) de preescolares venezolanos. Metodología: Se estudiaron 173 niños y se construyeron los indicadores antropométricos: talla/ edad, peso/ talla, área magra y área grasa del brazo, utilizando el patrón de Frisancho como referencia. Se creó y aplicó un instrumento para evaluar los hábitos alimentarios y el NAF. Resultados: El patrón de consumo fue bajo para el grupo de vegetales, frutas y grasas, $y$ alto para el consumo de cereales, carnes y lácteos. El NAF en $52 \%$ de los preescolares fue intenso y en $27 \%$ leve. Se encontró una asociación entre los hábitos alimentario, el NAF y el Índice de Sedentarismo (IS). Conclusiones: Los resultados sugieren que se debe implementar cambios desde la etapa preescolar, para corregir precozmente los inadecuados hábitos alimentarios, fomentar la actividad física y evitar los altos IS con la consecuente aparición de enfermedades degenerativas crónicas del adulto.

Palabras clave: preescolares; evaluación nutricionalantropométrica; hábitos alimentarios; actividad física.

Dirigir la correspondencia a:

Profesora

MsC Mariné Nava.

Departamento de Nutrición y Dietética.

Caracas, Venezuela.

Esq. De Miracielos a Hospital.

Edificio Sur 2. PH.

Oficina PH-05: Pquia. Santa Teresa.

Apartado Postal 1010

Caracas

Venezuela

Teléfono. $58(212) 8155492$

E-mail: bravo.marine@gmail.com 


\section{ANEXO 1}

Cuestionario de frecuencia de consumo de alimentos.

Marque con una X en la casilla correspondiente a la frecuencia de consumo de alimentos de su representado en el último mes por semana y por día.

\begin{tabular}{|c|c|c|c|c|c|c|c|c|}
\hline \multirow{2}{*}{\begin{tabular}{|l} 
\\
\\
Alimento
\end{tabular}} & \multirow{2}{*}{$\begin{array}{c}\text { FRECUENCIA DE } \\
\text { CONSUMO } \\
\\
\text { Cantidad }\end{array}$} & \multicolumn{3}{|c|}{$\begin{array}{c}\text { A LA } \\
\text { SEMANA }\end{array}$} & \multicolumn{3}{|c|}{ AL DÍA } & \\
\hline & & 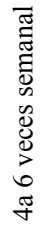 & 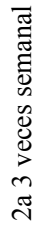 & 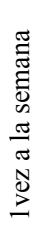 & $\begin{array}{l}\frac{\pi}{0} \\
\frac{\pi}{\pi} \\
0 \\
0 \\
0 \\
> \\
i \\
\pi \\
\pi\end{array}$ & $\begin{array}{c}\frac{\pi}{0} \\
\frac{\pi}{\pi} \\
0 \\
0 \\
0 \\
0 \\
\pi \\
\pi \\
\pi\end{array}$ & $\begin{array}{l}\frac{\pi}{\sigma} \\
\tilde{\sigma} \\
N \\
\mathbb{D}\end{array}$ & 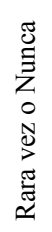 \\
\hline \multicolumn{9}{|l|}{ Cereales o verduras } \\
\hline Arepa o bollito & 1 pequeña & & & & & & & \\
\hline Pan & 1 rebanada & & & & & & & \\
\hline Empanada & 1 mediana & & & & & & & \\
\hline Panquecas & 1 pequeña & & & & & & & \\
\hline Verduras & Media taza & & & & & & & \\
\hline Arroz & Media taza & & & & & & & \\
\hline Pasta & Media taza & & & & & & & \\
\hline Granos & Media taza & & & & & & & \\
\hline Plátano & Un cuarto de unidad & & & & & & & \\
\hline Galletas & 3 unidades & & & & & & & \\
\hline \multicolumn{9}{|l|}{$\begin{array}{l}\text { Otro(especifique tipo y cantidad } \\
\text { consumida usualmente): }\end{array}$} \\
\hline \multicolumn{9}{|l|}{ Frutas } \\
\hline Melón & 1 taza & & & & & & & \\
\hline Naranja & 1 mediana & & & & & & & \\
\hline Guayaba & 1 mediana & & & & & & & \\
\hline Cambur & Media taza & & & & & & & \\
\hline Mango & Media unidad & & & & & & & \\
\hline Manzana & 1 mediana & & & & & & & \\
\hline Patilla & 1 taza & & & & & & & \\
\hline Parchita & 2 medianas & & & & & & & \\
\hline Piña & 1 rueda & & & & & & & \\
\hline \multicolumn{9}{|l|}{$\begin{array}{l}\text { Otro(especifique tipo y cantidad } \\
\text { consumida usualmente): }\end{array}$} \\
\hline \multicolumn{9}{|l|}{ Vegetales } \\
\hline Zanahoria & Media taza & & & & & & & \\
\hline Cebolla & Media taza & & & & & & & \\
\hline Tomate & Media taza & & & & & & & \\
\hline Lechuga & Media taza & & & & & & & \\
\hline Calabacín & Media taza & & & & & & & \\
\hline Pepino & Media taza & & & & & & & \\
\hline Repollo & Media taza & & & & & & & \\
\hline $\begin{array}{l}\text { Otro(especifique tipo y cantidad } \\
\text { consumida usualmente): }\end{array}$ & & & & & & & & \\
\hline
\end{tabular}




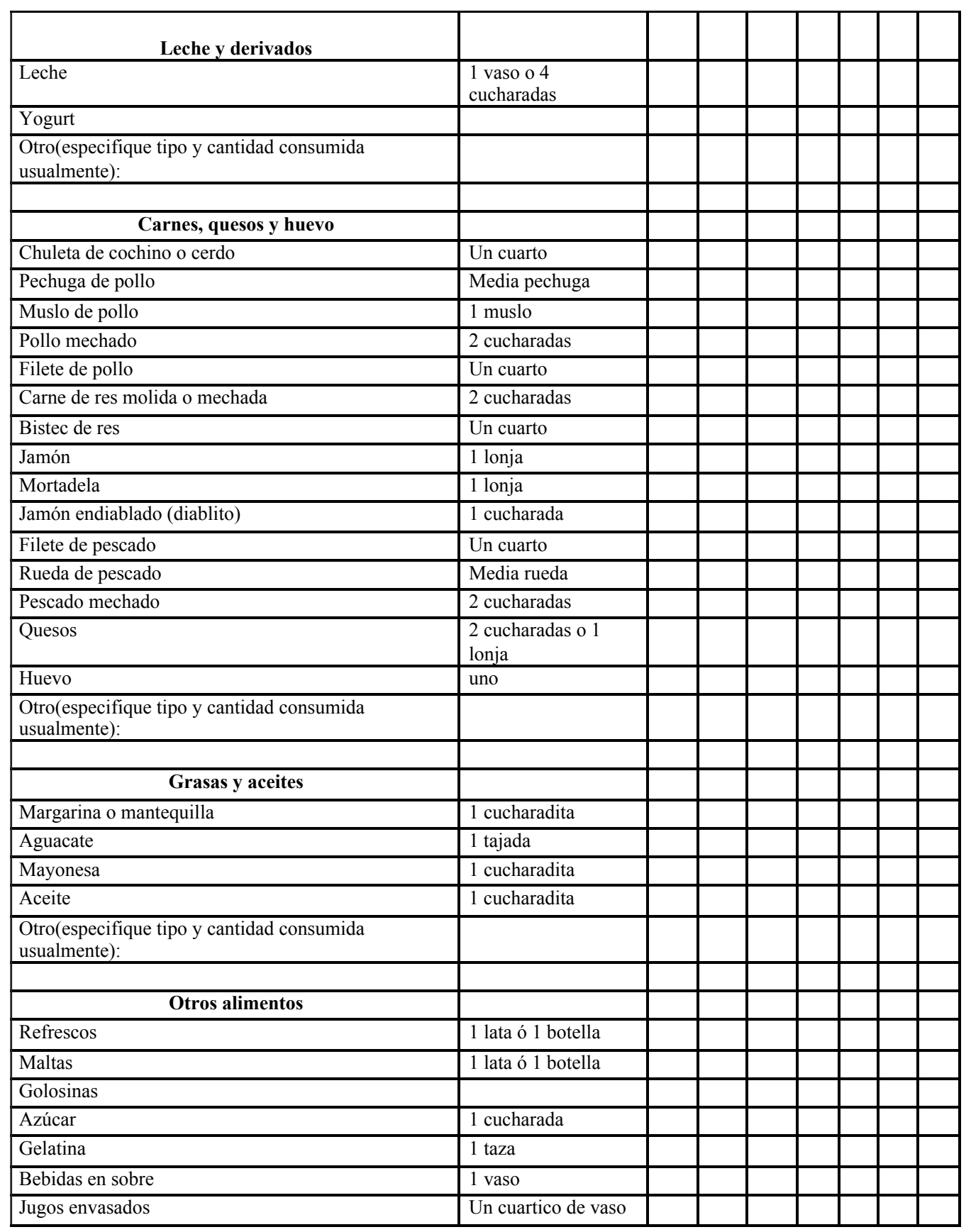

Si algún alimento que consume el niño no fue nombrado, especifique tipo y cantidad consumida usualmente

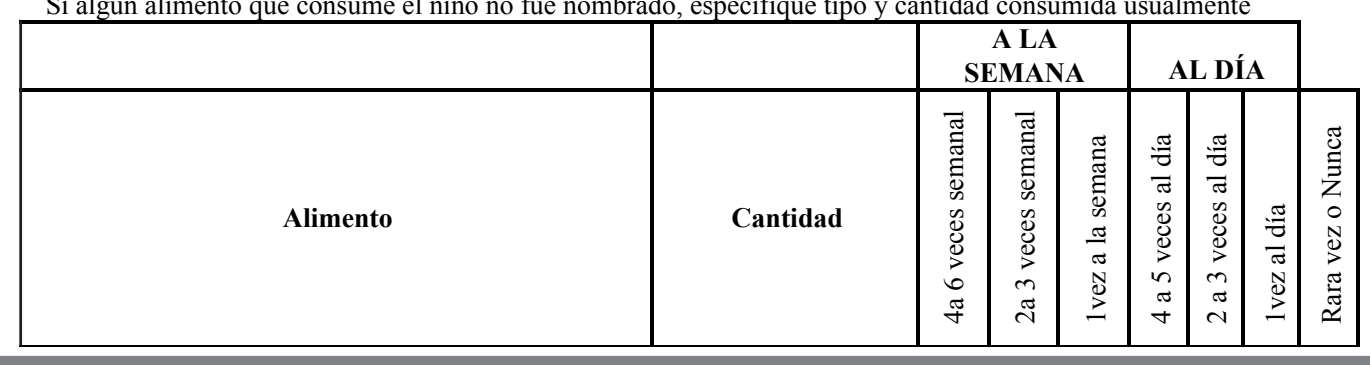




\section{ANEXO 2}

\section{Cuestionario de actividad física habitual.}

\section{Marque con una $x$ la opción correspondiente a la actividad física que su hijo o representado realiza:}

¿Cuál? Indique

¿Su hijo tiene algún impedimento físico para trotar, caminar, correr, etc.? Sí

No

¿Cuál? Indique

1. DURANTE LA SEMANA SU HIJO SUELE AYUDAR A:

\begin{tabular}{|l|l|c|c|c|c|}
\hline & Nunca & $\begin{array}{c}1-2 \text { veces a la } \\
\text { semana }\end{array}$ & $\begin{array}{c}3-4 \text { veces a la } \\
\text { semana }\end{array}$ & $\begin{array}{c}5-6 \text { veces a } \\
\text { la semana }\end{array}$ & $\begin{array}{c}\text { Más de 6 veces a la } \\
\text { semana }\end{array}$ \\
\hline Limpiar la casa & & & & & \\
\hline Lavar platos & & & & & \\
\hline
\end{tabular}

¿Cuántas horas al día su hijo realiza éstas actividades?

\begin{tabular}{|l|l|l|l|l|l|}
\hline & Menos de 1 hora & 1 hora & 2 horas & 3 horas & Más de 3 horas al día \\
\hline Limpiar la casa & & & & & \\
\hline Lavar platos & & & & & \\
\hline
\end{tabular}

2. ¿CUÁNTAS VECES A LA SEMANA, SIN INCLUIR LAS HORAS EN LA ESCUELA, SU HIJO:

\begin{tabular}{|c|c|c|c|c|c|}
\hline & Nunca & $\begin{array}{c}1-2 \text { veces a la } \\
\text { semana }\end{array}$ & $\begin{array}{l}\text { 3-4 veces a } \\
\text { la semana }\end{array}$ & $\begin{array}{l}5-6 \text { veces a } \\
\text { la semana }\end{array}$ & $\begin{array}{l}\text { Más de } 6 \text { veces a la } \\
\text { semana }\end{array}$ \\
\hline \multicolumn{6}{|l|}{ Juega en el parque } \\
\hline \multicolumn{6}{|l|}{ Juega } \\
\hline \multicolumn{6}{|l|}{ Corre } \\
\hline \multicolumn{6}{|c|}{$\begin{array}{l}\text { Camina sin peso } \\
\text { (morral, bulto, bolsas, } \\
\text { etc) }\end{array}$} \\
\hline \multicolumn{6}{|c|}{$\begin{array}{l}\text { Camina con peso } \\
\text { (morral, bulto, bolsas, } \\
\text { etc) }\end{array}$} \\
\hline \multicolumn{6}{|l|}{ Monta bicicleta } \\
\hline \multicolumn{6}{|l|}{ Baila } \\
\hline \multicolumn{6}{|l|}{ Estudia } \\
\hline Otras(especifique): & & & & & \\
\hline
\end{tabular}

¿Cuántas horas al día su hijo realiza éstas actividades? (sin incluir las horas que las realiza en la escuela)

\begin{tabular}{|l|l|l|l|l|l|}
\hline & ¿Cuántos minutos? & 1 hora & 2 horas & 3 horas & Más de 3 horas al día \\
\hline Juega en el parque & & & & & \\
\hline Juega & & & & & \\
\hline Corre & & & & & \\
\hline Camina sin peso & & & & & \\
\hline $\begin{array}{c}\text { Camina con peso (morral, } \\
\text { bulto, bolsas, etc) }\end{array}$ & & & & & \\
\hline Monta bicicleta & & & & & \\
\hline
\end{tabular}




\begin{tabular}{|l|l|l|l|l|l|}
\hline Baila & & & & & \\
\hline Estudia & & & & & \\
\hline Otras(especifique): & & & & & \\
\hline
\end{tabular}

Practica su hijo algún deporte: Sí ¿Cuál o cuáles?

¿Cuántas veces a la semana?

¿Cuántas horas?

II. ¿CUÁNTAS HORAS?

\begin{tabular}{|c|c|c|c|c|c|c|}
\hline & Ninguna & $\begin{array}{l}1 \text { a } 2 \\
\text { horas }\end{array}$ & $\begin{array}{l}2 \text { a } 4 \\
\text { horas }\end{array}$ & $\begin{array}{l}4 \text { a } 6 \\
\text { horas }\end{array}$ & $\begin{array}{l}6 \text { a } 8 \\
\text { horas }\end{array}$ & Más de 8 horas \\
\hline $\begin{array}{l}\text { ¿Su hijo ve televisión en un } \\
\text { día de semana? }\end{array}$ & & & & & & \\
\hline $\begin{array}{l}\text { ¿Su hijo ve televisión en un } \\
\text { día del fin de semana? }\end{array}$ & & & & & & \\
\hline $\begin{array}{l}\text { ¿Su hijo se dedica a jugar con } \\
\text { juegos de video en un día de } \\
\text { semana? }\end{array}$ & & & & & & \\
\hline $\begin{array}{l}\text { ¿Su hijo se dedica a jugar con } \\
\text { juegos de video en un día del } \\
\text { fin de semana? }\end{array}$ & & & & & & \\
\hline $\begin{array}{l}\text { ¿Su hijo duerme en un día de } \\
\text { semana, desde que se } \\
\text { acuesta en la noche hasta } \\
\text { levantarse en la mañana? }\end{array}$ & & & & & & \\
\hline $\begin{array}{l}\text { ¿Su hijo duerme en un día del } \\
\text { fin de semana, desde que se } \\
\text { acuesta en la noche hasta } \\
\text { levantarse en la mañana? }\end{array}$ & & & & & & \\
\hline $\begin{array}{l}\text { ¿Su hijo duerme en un día de } \\
\text { semana, además de las horas } \\
\text { de sueño de la noche? }\end{array}$ & & & & & & \\
\hline $\begin{array}{l}\text { ¿Su hijo duerme en un día del } \\
\text { fin de semana, además de las } \\
\text { horas de sueño de la noche? }\end{array}$ & & & & & & \\
\hline
\end{tabular}

\section{BIBLIOGRAFÍA}

1. Aliño M, Navarro R, López J, Pérez I. La edad preescolar como momento singular del desarrollo humano. Rev Cubana Pediatr 2007; 79 (4).

2. Solano L, Velásquez E, Naddaf G, Páez M. Patrón de lípidos en preescolares de bajos recursos socioeconómicos (Valencia, Venezuela). Acta Cient Venez. 2003; 54(4):248-53.

3. Del Real S, Sánchez J, Barón M, Díaz N, Solano L, Velásquez E, et al. Estado nutricional en niños preescolares que asisten a un jardín de infancia pú- blico en Valencia, Venezuela. Arch Latinoam Nutr 2007; 57(3): 248-54.

4. Macmillan N. Evaluación del estado nutricional, hábitos de alimentación y actividad física en escolares de $1^{\circ}$ básico de Isla de Pascua. Rev Chil Nutr 2005; 32(3):232-7.

5. Haidar J, Abate G, Kogi W, Sorensen P. Risk factors for children under-nutrition with a human rights edge in rural vollages of North Wollo, Etiopía. East Afr Med 2005; 82(12):626-30.

6. Velásquez M, Salazar G, Vio F, Díaz N, Ansían 
A. Validación de ecuaciones antropométricas para evaluar composición corporal en niños preescolares chilenos Rev Med Chil 20g8; 136:433-41.

7. Stanojevic S, Kain J, Uauy R. Secular and seasonal trends in obesity in Chilean preschool_children, 1996-2004. J Pediatr Gastroenterol Nutr 2008; 47(3):339-43.

8. Organización Mundial de la Salud. Estrategia Mundial sobre Régimen Alimentario, Actividad física y Salud. 57 ${ }^{\mathrm{a}}$ Asamblea Mundial de Salud. 2004.

9. Asociación Médica Mundial (WMA). Declaración de Helsinki: Principios básicos, operacionales y pautas. Helsinki (Finlandia); 1964.

10. Lohman TG, Roche F, Martorell R. Anthropometric Standarization Manual. Kinetics Books, Illinois. 1988.

11. Herrera HA, Hernández R, Rodríguez N. Manual de procedimientos y técnicas antropométricas. 1era ed, Caracas; 2005.

12. Jelliffe E, Jellife D. The arm circunference as a public health index of protein-calorie malnutrition of early childhood. J Trop Pediatr 1969; 32:1527-30.

13. Frisancho R. Anthropometric Standars for the Assesment of Growth and Nutritional Status.The University of Michigan Press, Michigan, 1990.

14. Prista A, Maia J, Marques A. An empirical validation of an instrument to measure habitual physical activity in students from Maputo, Mozambique. Am L Hum Biol 2000; 12: 437- 46.

15. Instituto Nacional de Nutrición. Alimentación y Nutrición en grupos vulnerables. Alimentación del menor de seis años. Serie: Nutrición Comunitaria. Sección B. Caracas-Venezuela.1998.

16. Ainsworth B, Haskell W, Whitt M, Irwin M, Swartz A, Strath S, et al. Compendium of physical activities: an update of activity codes and MET intensities. Med Sci Sports Exerc 2000; 32: 498-516.

17. García M. Cálculos de los requerimientos nutricionales. 1era ed. Cania editor, Caracas; 1999.

18. Torun B, Davies P, Livingstone M, Paolisso M, Sackett R, Spurr G, et al. Energy requirements and dietary recommendations for children and adolescent 1 to 18 years old. Eur J Clin Nutr 1996; 50 Supl 1:S37-81.

19. Martínez M, Martínez J, Hu F, Gibney, Kearney J. Physical inactivity, sedentarism lifestyle and obesity in the European Union. Int J Obes 1999; 23:1-10.

20. American Academy of Pediatrics. Children, adolescents, and television. Pediatrics 2001; 107
(2):423-26.

21. Vásquez F, Salazar G. Patrón de actividad física en un grupo de preescolares obesos asistentes a jardines infantiles de Junji, evaluado con sensor de movimiento. Rev Chil Nutr 2005; 32(2):110-7.

22. Moreira P, Santos S, Padrao P, Cordeiro T, Bessa $\mathrm{M}$, Valente $\mathrm{H}$, et al. Food Patterns According to Sociodemographics, Physical Activity, Sleeping and Obesity in Portuguese Children. Int J Environ Res Public Health 2010:7(3) : 1121-38

23. Wosje-K Khowry $\mathrm{H}$, Claytor R, Copeland K, Hornung R, Daniels S, et al. Dietary patterns associated with fat and bone mass in young children. Am J Clin Nutr 2010; 92(2): 294-303.

24. Kain B, Lera L, Rojas J, Uauy R. Obesidad en preescolares de la Región Metropolitana de Chile. Rev Med Chil 2007; 135: 63-70.

25. Instituto Nacional de Nutrición -Sistema de Vigilancia Alimentaria Nutricional (INN-SISVAN). Anuario del sistema alimentario de vigilancia nutricional Componente menores de 15 años. Unidad de Nutrición del Dtto. Capital. Caracas-Venezuela, 2004.

26. Van Dam R, Willett W, Rimm E, Stampfer M, Hu F. Fat and Meat Intake in Relation to Risk of Type 2 Diabetes in Men. Diabetes Care 2002; 25: 417-24.

27. Davies P. Diet composition and body mass index in pre-schoolchildren. Eur J Clin Nutr 1997; 51:443-8.

28. Fanjiang G, Kleinman R Nutrition and performance in children. Curr Opin Clin Nutr Metab Care 2008; 10(3):342-7.

29. Vásquez F, Cardona O, Andrade M, Salazar G. Balance de energía, composición corporal y actividad física en preescolares eutróficos y obesos. Rev Chil Pediatr 2005; 76(3):266-74.

30. Reilly, J. Physical activity, sedentary behaviour and energy balance in the preschoolchild. opportunities for early obesity prevention. Proc Nutr Soc 2008; 67(3) $\cdot 317-25$

31. Lioret S, Maire B, Volatier J, Charles M Child overweight in France and its relationship with physical activity sedentary hehaviour and socioeconomic status. Eur J Clin Nutr 2007; 61(4):509-16.

32. Giraldo D, Poveda E, Forero V, Mendivil C, Castro L. Actividad física autoreportada, comparación con indicadores antropométricos de grasa corporal en un grupo de escolares de Bogotá y de cinco departamentos del centro oriente, Colombia 2000 - 2002. Biomédica 2008; 28:386-95. 